\title{
Synthesis of ultrathin rhenium disulfide nanoribbons using nano test tubes
}

\author{
Luke T. Norman ${ }^{1}$, Johannes Biskupek ${ }^{2}$, Graham A. Rance ${ }^{3}$, Craig T. Stoppiello ${ }^{3}$, Ute Kaiser ${ }^{2}$, and \\ Andrei N. Khlobystov ${ }^{1}(\Delta)$ \\ ${ }^{1}$ School of Chemistry, University of Nottingham, Nottingham, NG7 2RD, UK \\ ${ }^{2}$ Central Facility for Electron Microscopy, Ulm University, Albert Einstein-Allee 11, Ulm 89081, Germany \\ ${ }^{3}$ Nanoscale and Microscale Research Centre, University of Nottingham, Nottingham, NG7 2RD, UK
}

(C) The Author(s) 2021

Received: 20 April 2021 / Revised: 1 June 2021 / Accepted: 2 June 2021

\begin{abstract}
The synthesis of ultrathin rhenium disulfide $\left(\mathrm{ReS}_{2}\right)$ nanoribbons within single-walled carbon nanotubes (SWNTs) has been established. Dirhenium decacarbonyl complex is encapsulated into the SWNTs to provide a source of confined rhenium atoms, which readily react with iodine to form discrete $n m$-sized clusters of rhenium iodide $\left[\mathrm{Re}_{6} \mathrm{I}_{14}\right]^{2-}$ embedded in the nanotubes. The final step of the synthesis is accomplished by admitting hydrogen sulfide gas into nano test tubes, yielding twisted nanoribbons of rhenium disulfide encapsulated in carbon nanotubes, $\mathrm{ReS}_{2} @$ SWNTs. The width, structure, and composition of rhenium disulfide nanoribbons are strictly controlled by the extreme confinement of the host-SWNT. A holistic analytical approach combining complementary imaging and analysis methods is used at each synthetic step to elucidate the structure and composition of the guest material and reveal the role of the SWNT contributing towards the electronic interactions with encapsulated inorganic structures. As ReS $\mathrm{S}_{2}$ nanoribbons are expected to retain the electronic properties of the bulk material, such as direct bandgap, the low dimensional form of this material can be of interest for use in nanoscale electronic devices.
\end{abstract}

KEYWORDS

nanotubes, nanoribbons, rhenium, confinement, microscopy, spectroscopy

\section{Introduction}

Rhenium(IV) disulfide $\left(\mathrm{ReS}_{2}\right)$ has gained significant recent attention following the discovery that it, uniquely amongst the transition metal dichalcogenides (TMDs), possesses layerindependent optical, electrical, and vibrational properties, as well as exhibiting in-plane anisotropicity [1-3]. When exfoliated to a monolayer it retains its electronic properties as a direct bandgap semiconductor; however, the bandgap does slightly increase from $1.5 \mathrm{eV}$ for the bulk material to $1.6 \mathrm{eV}$ for the monolayer [4]. This is in stark contrast to most other TMDs whose physical properties are layer-dependent and, when exfoliated to a monolayer, undergo an indirect to direct bandgap transition resulting in photoluminescence $[5,6]$. The layered $\mathrm{ReS}_{2}$ material has a distorted $1 \mathrm{~T}$ structure [7] which causes the in-plane anisotropicity of the layers, as demonstrated by Raman spectroscopy [8].

Various $\mathrm{ReS}_{2}$-based nanomaterials, such as nanotubes, have been synthesised previously [9]. These were formed upon reaction of a Re-containing solution with $\mathrm{H}_{2} \mathrm{~S}$ at the surface of multi-walled carbon nanotubes, which act as a template. Colloidal nanoparticles with an average diameter of $5.5 \mathrm{~nm}$ have also been formed [10]. Nanowires with a diameter of $180 \mathrm{~nm}$ were synthesised by An et al. using a chemical vapour deposition (CVD) technique [11]. $\mathrm{ReS}_{2}$ nanoribbons were synthesised by $\mathrm{He}$ et al. also by a CVD process; the width of the nanoribbons were approximately $50 \mathrm{~nm}$ and the lengths ranged from 10 to $30 \mu \mathrm{m}[12]$. However, the structure and composition of ultrathin $\mathrm{ReS}_{2}$ nanoribbons (1.3-3.8 nm) have only been studied computationally, where they were found to have a direct bandgap which is only weakly dependent on the nanoribbon width [13].

Single-walled carbon nanotubes (SWNTs) are electrically conducting [14] hollow tubular structures which were first identified in $1993[15,16]$. They have diameters in the range of $0.8-2.0 \mathrm{~nm}$ which therefore restrict the materials that can fit inside, as well as providing a mechanism to template the growth of novel one-dimensional (1D) structures [17, 18]. They have previously been shown to be suitable containers for a variety of chemical species, such as fullerenes [19, 20], halides [21-23], and metal oxides [24, 25]. Moreover, entrapping guest-species within the SWNT channel allows the structures and compositions of the encapsulated materials to be studied with atomic precision using electron microscopy coupled with spectroscopic techniques [26].

Inorganic reactions can also be conducted within SWNTs by encapsulating precursor molecules followed by a thermal activation of the system [27]. SWNTs offer strict control over the structure and composition of the 1D materials formed and have been applied as a template for a wide range of ultrathin species, such as sulfur-terminated graphene nanoribbons [28], and metal sulfides $[29,30]$. It has also been shown that SWNTs can act as a nanoreactor transferring electrons to and from guest species in order to stabilise them within the interior 
channel [31]. This was shown previously by Botos et al. who synthesised $\mathrm{MoS}_{2}$ and $\mathrm{WS}_{2}$ nanoribbons using metal carbonyls as the precursors [31]. The SWNTs were found to donate electron density to iodine forming polyiodide ions, which reacted with the metal carbonyl precursors to form $\left[\mathrm{M}_{6} \mathrm{I}_{14}\right]^{2-}$ clusters that were stabilised by Coulombic attraction with the resultant electron deficient SWNTs. This method has been shown to be suitable for the formation of $1 \mathrm{D}$ molybdenum and tungsten disulfides, and can be utilised for materials with other remarkable properties at the nanoscale, such as $\mathrm{ReS}_{2}$. Previously $\mathrm{ReS}_{2}$ was grown on the exterior of carbon nanotubes and has been tested as an anode material for Li-ion batteries and shown high capacitance and capacity retention after 100 cycles [32].

Herein we report, the synthesis of ultrathin $\mathrm{ReS}_{2}$ nanoribbons with diameters in the range of 1-2 $\mathrm{nm}$ which have only been previously studied theoretically. The synthetic route utilises dirhenium decacarbonyl $\left(\operatorname{Re}_{2}(\mathrm{CO})_{10}\right)$ as the source of rhenium atoms, with rhenium iodide the intermediate in the final formation of $\mathrm{ReS}_{2}$. The use of the nano test tube not only provides a mechanism for the directional growth of rhenium disulfide, but also a means to study the atomic structure. A combination of microscopy and spectroscopy was used to probe the structure and composition of the materials in SWNTs at each stage of the stepwise synthesis. The ability to understand and control the inorganic transformations at the nanoscale enables the rational design of functional nanomaterials for a variety of applications.

\section{Experimental}

Materials: SWNTs (P2-SWNTs, arc discharge, Carbon Solutions, USA) were refluxed in $3 \mathrm{M}$ nitric acid for $2 \mathrm{~h}$ and then annealed at $600{ }^{\circ} \mathrm{C}$ for $17 \mathrm{~min}$ to remove their end caps and any amorphous carbon present, resulting in a $50 \%$ weight loss observed. Dirhenium decacarbonyl (Sigma Aldrich), iodine (Sigma Aldrich), and hydrogen sulfide (Fisher Scientific) were used as supplied.

Insertion of dirhenium decacarbonyl into SWNTs: Freshlyopened SWNTs $(10 \mathrm{mg})$ and dirhenium decacarbonyl $(20 \mathrm{mg}$, $0.031 \mathrm{mmol}$ ) were sealed in a Pyrex ampoule under vacuum $\left(10^{-5} \mathrm{mbar}\right)$ and heated to $150{ }^{\circ} \mathrm{C}$ for 3 days. The ampoule was then placed in an ice bath to cease the sublimation process and then washed with tetrahydrofuran to remove any excess material from the exterior of the SWNTs.

Reaction of $\operatorname{Re}_{2}(\mathrm{CO})_{10} @ S W N T$ with iodine: $\operatorname{Re}_{2}(\mathrm{CO})_{10} @ S W N T$ $(10 \mathrm{mg}, 0.016 \mathrm{mmol})$ and a large excess of iodine were sealed in a Pyrex ampoule under vacuum $\left(10^{-5} \mathrm{mbar}\right)$ and heated to $120{ }^{\circ} \mathrm{C}$ for 3 days. The ampoule was then placed in an ice bath to cease the sublimation process. The resultant material was then placed in a Pyrex ampoule and sealed under an inert argon atmosphere $\left(0.7\right.$ bar) and heated to $500{ }^{\circ} \mathrm{C}$ for $1 \mathrm{~h}$ to form $n\left[\operatorname{Re}_{6} \mathrm{I}_{14}{ }^{2-}\right] @ \mathrm{SWNT}^{2 n+}$.

Reaction of $n\left[\operatorname{Re}_{6} \mathrm{I}_{14}{ }^{2-}\right] @ S W N T^{2 n+}$ with hydrogen sulfide: $n\left[\mathrm{Re}_{6} \mathrm{I}_{14}{ }^{2-}\right] @ \mathrm{SWNT}^{2 n+}$ was loaded onto a quartz boat and placed in the centre of a tube furnace and exposed to a flow of argon gas for $1 \mathrm{~h}(15 \mathrm{~mL} / \mathrm{min})$, followed by a flow of hydrogen sulfide $(15 \mathrm{~mL} / \mathrm{min})$. The furnace was then heated to $550{ }^{\circ} \mathrm{C}$ for $2 \mathrm{~h}$ under the flow of hydrogen sulfide $(15 \mathrm{~mL} / \mathrm{min})$ and then left to cool to room temperature under a flow of argon (15 mL/min).

Electron microscopy and energy-dispersive X-ray analysis: The samples were dispersed in isopropyl alcohol using an ultrasonic bath and drop cast onto lacey carbon copper transmission electron microscopy (TEM) grids (Agar Scientific). High-resolution TEM (HR-TEM) imaging was performed on a JEOL 2100F FEG-TEM microscope operated at $200 \mathrm{kV}$. Aberration-corrected HRTEM (AC-HRTEM) was performed at the University of Ulm using the dedicated $\mathrm{C}_{\mathrm{C}} / \mathrm{C}_{\mathrm{s}}$ corrected SALVE (sub-Ångström low voltage electron) TEM operating a $60 \mathrm{kV}$ [33]. Energy-dispersive X-ray (EDX) spectra were acquired using an Oxford Instruments INCA X-ray microanalysis system.

Raman spectroscopy: Micro Raman spectroscopy was performed using a Horiba Jobin Yvon LabRAM HR Raman microscope. Spectra were acquired using either a 532 or $660 \mathrm{~nm}$ laser (at $\sim 0.2 \mathrm{~mW}$ power), a $100 \times$ objective and a $200 \mu \mathrm{m}$ confocal pinhole. To simultaneously scan a range of Raman shifts, a 600 lines $\mathrm{mm}^{-1}$ rotatable diffraction grating along a path length of $800 \mathrm{~mm}$ was employed, conferring a spectral resolution better than 1.9 and $1.2 \mathrm{~cm}^{-1}$ for the 532 and $660 \mathrm{~nm}$ lasers, respectively. Spectra were detected using a Synapse CCD detector (1,024 pixels) thermoelectrically cooled to $-60^{\circ} \mathrm{C}$. Before spectra collection, the instrument was calibrated using the zero-order line and a standard $\mathrm{Si}(100)$ reference band at $520.7 \mathrm{~cm}^{-1}$. For single point measurements, spectra were acquired over a minimum range $125-3,400 \mathrm{~cm}^{-1}$ with an acquisition time of $15-120 \mathrm{~s}$ and 2 accumulations to automatically remove the spikes due to cosmic rays and improve the signal to noise ratio. Spectra were collected from at least three random locations and averaged to give a mean spectrum.

X-ray photoelectron spectroscopy (XPS): Samples were analysed using the Kratos AXIS ULTRA with a mono-chromated $\mathrm{Al} \mathrm{k} \alpha \mathrm{X}$-ray source $(1,486.6 \mathrm{eV})$ operated at $10 \mathrm{~mA}$ emission current and $12 \mathrm{kV}$ anode potential (120 W.) Spectra were acquired with the Kratos VISION II software. A charge neutralizer filament was used to prevent surface charging. Hybrid slot mode was used measuring a sample area of approximately $300 \mu \mathrm{m} \times 700 \mu \mathrm{m}$. The analysis chamber pressure was better than $5 \times 10^{-9}$ mbar. Three areas per sample were analysed. A wide scan was performed at low resolution (Binding energy range 1,400 to $-5 \mathrm{eV}$, with pass energy $80 \mathrm{eV}$, step $0.5 \mathrm{eV}$, and sweep time $20 \mathrm{~min}$ ). High resolution spectra at pass energy of $20 \mathrm{eV}$, step of $0.1 \mathrm{eV}$, and sweep time of $10 \mathrm{~min}$ each were also acquired for photoelectron peaks from the detected elements and these were used to model the chemical composition. The spectra were charge corrected to the $\mathrm{C} 1$ s peak set to $284.5 \mathrm{eV}$.

TEM simulations: TEM image simulation was carried out using the multislice program QSTEM. QSTEM uses the Dirac-Fock scattering potential of Rez et al. [34]. A fixed number of 20 slices per nanotube were chosen and images were calculated with a sampling of $0.125 \AA$ per pixel. The defocus parameter $d_{f}$ and the aberration coefficients $C_{S}, A_{1}$, and $B_{2}$ were matched to the imaging conditions in the specific experiment and the convergence angle was fixed at $0.5 \mathrm{mrad}$. The effect of limited electron dose was emulated by applying noise to the calculated images using a custom-made Monte-Carlo program exploiting the Poisson statistics of electrons. Structural models of SWNTs were built using a custom-made program taking into account different nanotube chiralities, to be matched closely with experimental images. The structural models of the inorganic nanomaterials are based on scaled bulk structures.

\section{Results and discussion}

\subsection{Stepwise synthesis}

To prepare ultrathin rhenium iodide and sulfide materials a stepwise synthesis was utilised (Fig. 1). Dirhenium decacarbonyl was selected as the precursor due to its low sublimation temperature and labile carbonyl ligands; this precursor has been used before to prepare Re nanoparticles inside SWNTs by $\mathrm{Cao}$ et al. [35]. An iodide is used as the intermediate as it 


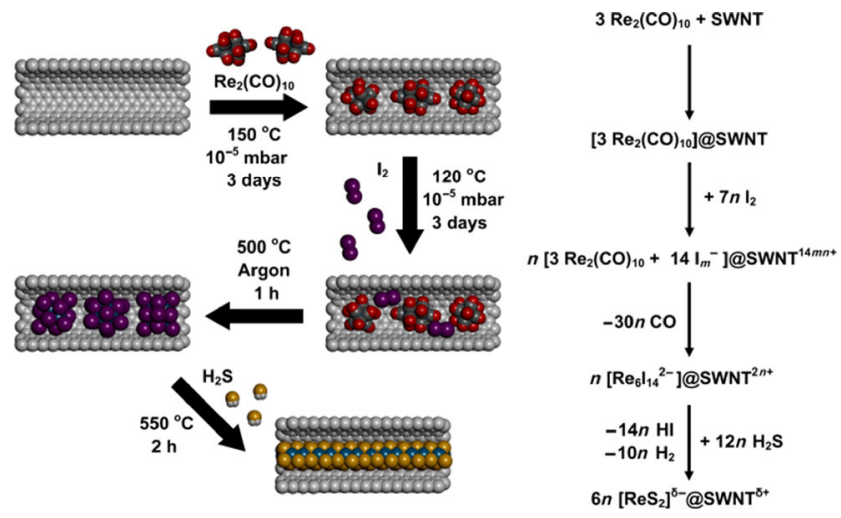

Figure 1 The synthesis of ReS $@ S W N T$ formed by the encapsulation and reaction of Re clusters. The clusters are first reacted to form rhenium iodide clusters using iodine and then a subsequent inorganic transformation takes place to form rhenium disulfide nanoribbons using a suitable reactant (left). A balanced chemical reaction demonstrating the charge transfer between nanotube and guest-molecules is also shown (right). $\mathrm{I}_{m}{ }^{-}$represents polyiodide ions $\left(\mathrm{I}_{3}{ }^{-}\right.$and $\left.\mathrm{I}_{5}{ }^{-}\right)$, and in the balanced equation $m$ is arbitrarily given the value 1 but this will change depending on exact stoichiometries produced.

offers the opportunity to examine entrapped forms of rhenium iodide which have not been previously studied before at the nanoscale. The final step of the synthesis involves the reaction of the iodide species with hydrogen sulfide to form the $\mathrm{ReS}_{2}$ nanoribbons.

\subsection{Insertion of the carbonyl complex}

The insertion of the dirhenium decacarbonyl in nanotubes was confirmed by $200 \mathrm{kV}$ TEM imaging, which displayed clusters with an average diameter of $0.6 \mathrm{~nm}$ (Fig. 2(a)), separated by distances above $3 \mathrm{~nm}$. A small number of the clusters grew during imaging to over $1 \mathrm{~nm}$ in size within the nanotube and thus are larger than expected and due to electron beaminduced ejection of carbonyl ligands, resulting in $\mathrm{Re}_{2}$ units that bond together forming rhenium nanoclusters [35]. EDX analysis of a bundle of cluster-filled nanotubes shows the presence of $\mathrm{Re}, \mathrm{C}$, and $\mathrm{O}$ corresponding to the elements in the carbonyl (Fig. 2(b)). Quantitative EDX analysis using a focused e-beam positioned over a bundle of 6-7 filled nanotubes suspended over a hole gives a $\mathrm{Re}: \mathrm{C}$ atomic ratio of 1:85. XPS was used to determine the binding state of the rhenium species in each step of the synthesis (Fig. 2(c)). The peaks for rhenium at 42.1 and $44.5 \mathrm{eV}$ were assigned to $\operatorname{Re} 4 \mathrm{f}_{7 / 2}$ and $4 \mathrm{f}_{5 / 2}$, respectively (Fig. S1(a) in the Electronic Supplementary Material (ESM)). The peak energies are higher than rhenium metal, which is explained by the presence of five $\pi$-acceptor carbonyl ligands per metal centre in $\mathrm{Re}_{2}(\mathrm{CO})_{10}$ which increase the binding energy [36]. The $\mathrm{C} 1 \mathrm{~s}$ peak at $284.5 \mathrm{eV}$ is mostly contributed from the SWNT hosts and not the guest carbonyls and the $\mathrm{O} 1 \mathrm{~s}$ peak around $532 \mathrm{eV}$ consists of multiple components arising mainly from the various $\mathrm{C}-\mathrm{O}$ bonds on the exterior of the SWNTs (Figs. S1(b) and S1(c) in the ESM). Van der Waals interactions are shown to be the main driving force for insertion of the carbonyl clusters into SWNTs as demonstrated by Raman spectroscopy (Fig. 2(d) and Table S1 in the ESM). The G-band of nanotubes did not shift for $\operatorname{Re}_{2}(\mathrm{CO})_{10} @ S W N T$ compared to empty SWNTs in the $660 \mathrm{~nm}$ Raman spectra, thus indicating no electronic communication between the host and guest.

\subsection{Reaction to form the iodide species}

Iodine was then inserted into the SWNTs under vacuum from the gas phase at $120^{\circ} \mathrm{C}$. The $532 \mathrm{~nm}$ Raman spectra, resonantly enhancing semiconducting SWNTs, showed a blue shift of the G-band indicating electron transfer from the SWNTs to iodine forming polyiodide ions $\left(\mathrm{I}_{m}{ }^{-}\right)$. This is further evidenced by the presence of low-frequency bands at 106 and $175 \mathrm{~cm}^{-1}$ which have been shown previously to be the result of polyiodide formation and are attributed to $\mathrm{I}_{3}^{-}$and $\mathrm{I}_{5}{ }^{-}$, respectively; however, it should be noted that there is strong overlap of the $\mathrm{I}_{5}{ }^{-}$band with the radial breathing mode of the SWNTs (Fig. S2 in the ESM) [37]. The G-band in the $660 \mathrm{~nm}$ Raman spectra, in resonance with metallic SWNTs, was blue shifted to a larger extent $\left(+10.6 \mathrm{~cm}^{-1}\right.$, Fig. $2(\mathrm{~d})$ and Table $\mathrm{S} 1$ in the ESM) and a
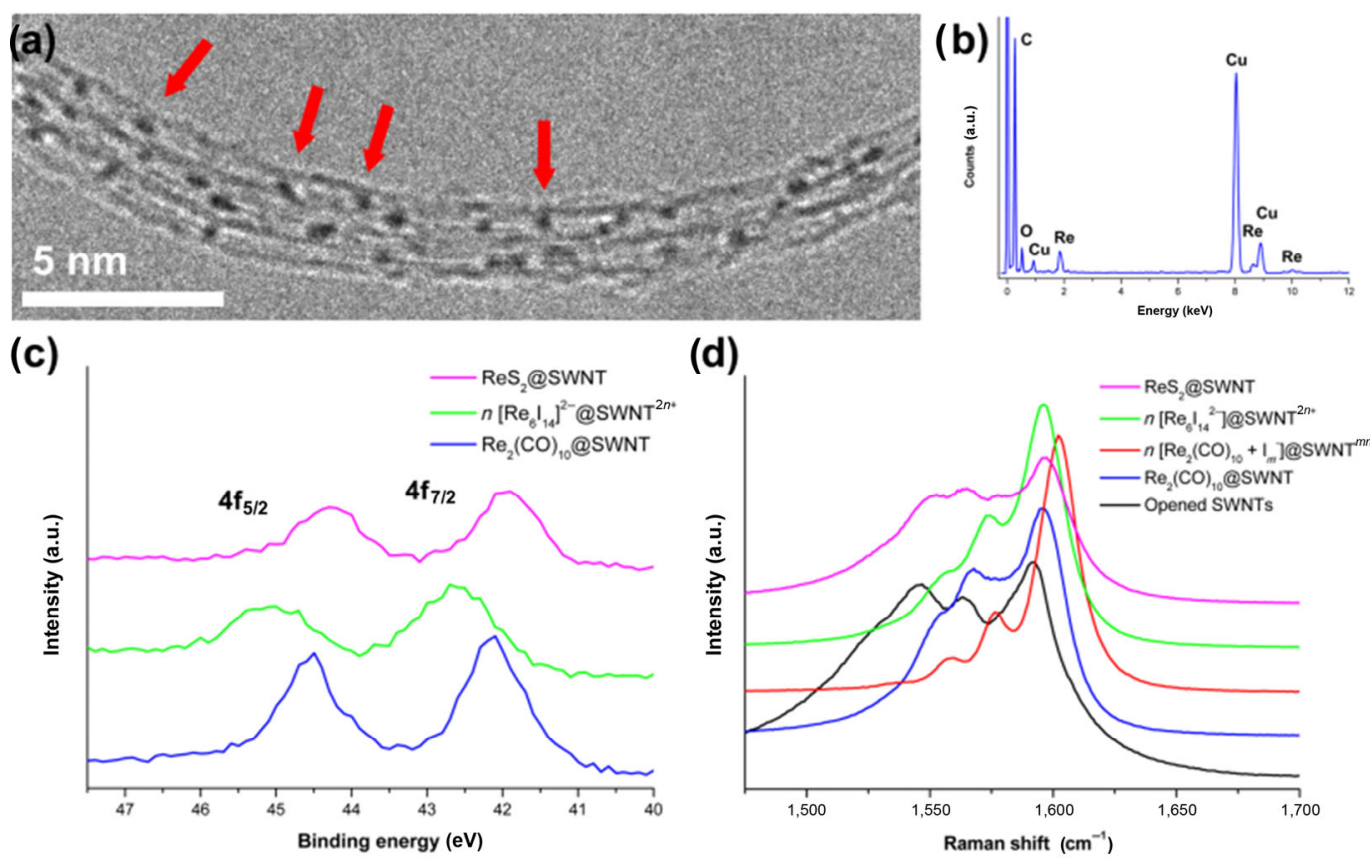

Figure 2 (a) $200 \mathrm{kV}$ TEM image of clusters formed from dirhenium decacarbonyl in a bundle of three SWNTs. A selection of the encapsulated clusters is indicated by red arrows superimposed on the image. (b) EDX spectrum identifying the presence of Re, $\mathrm{C}$, and $\mathrm{O}$. The presence of copper peaks is due to the TEM grid support. (c) XPS spectra overlay showing the $\operatorname{Re} 4 \mathrm{f}_{5 / 2}$ (left) and $4 \mathrm{f}_{7 / 2}$ (right) peaks of each material. (d) Raman spectra overlay focused on the G-band region for each material using $660 \mathrm{~nm}$ laser source exciting metallic SWNTs. 
change in the peak shape was recorded indicating a metallic to semiconducting transition due to Fermi level shift and band gap opening in the metallic SWNTs upon charge transfer [38].

The $n\left[\operatorname{Re}_{2}(\mathrm{CO})_{10}+\mathrm{I}_{m}{ }^{-}\right] @ S W N T^{m n+}$ system was subsequently heated under inert atmosphere to react together the encapsulants. Aberration-corrected high-resolution TEM (AC-HRTEM) imaging at $60 \mathrm{kV}$ after the thermally-activated reaction showed the presence of discrete tightly packed clusters of $\left[\operatorname{Re}_{6} \mathrm{I}_{14}\right]^{2-}$ $0.7 \mathrm{~nm}$ in diameter within the SWNT channel only allowing minimal translation motion; however, the clusters appear to rotate freely allowing various projections to be observed (Figs. 3(a)-(c)). Upon formation of the clusters, the G-band of metallic SWNTs red-shifts compared to when iodine is initially inserted into the SWNTs. However, it is still blue shifted compared to empty SWNTs, suggesting a positive charge on the nanotubes and negatively charged species are present within the SWNTs (Fig. $2(\mathrm{~d})$ ). The rhenium $4 \mathrm{f}_{7 / 2}$ and $4 \mathrm{f}_{5 / 2}$ peaks, observed in the XPS, are shifted to higher binding energies compared to the carbonyl, at 42.7 and $45.0 \mathrm{eV}$, respectively (Fig. 2(c) and
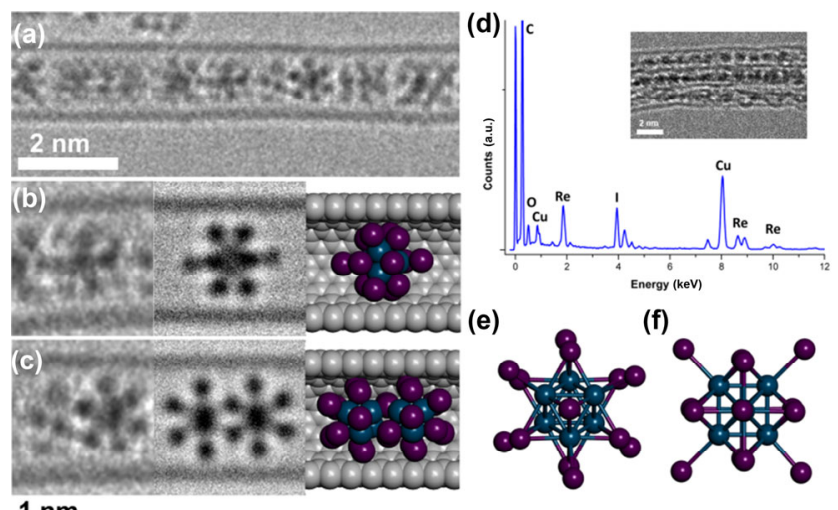

$1 \mathrm{~nm}$

Figure 3 (a) $60 \mathrm{kV}$ AC-HRTEM image of $n\left[\mathrm{Re}_{6} \mathrm{I}_{14}{ }^{2-}\right] @ \mathrm{SWNT}^{2 n+}$. (b) and (c) Experimental TEM images, TEM image simulations, and structural models of $n\left[\operatorname{Re}_{6} \mathrm{I}_{14}{ }^{2-}\right] @ S W N T^{2 n+}$ and $\left[\mathrm{Re}_{6} \mathrm{I}_{12}\right]_{2} @ S W N T$ respectively. (d) EDX spectrum of $n\left[\mathrm{Re}_{6} \mathrm{I}_{14}{ }^{2-}\right] @ S W N T^{2 n+}$ identifying both Re and I in an atomic 6:14 ratio. The presence of copper is due to the TEM grid support. Inset is a $200 \mathrm{kV}$ TEM image showing the packing of the clusters in the SWNTs. (e) and (f) Structural diagrams of $\left[\operatorname{Re}_{6} \mathrm{I}_{14}\right]^{2-}$ cluster viewed along the $C_{3}$ and $C_{4}$ rotational axis of the molecule, respectively.
Fig. S3(a) in the ESM). The iodine $3 \mathrm{~d}$ peaks were located at 619.6 and $631.1 \mathrm{eV}$ for the $5 / 2$ and $3 / 2$ components, which are expected for such species. EDX analysis identified the presence of both rhenium and iodine in an atomic ratio of approx. 6:14 (Fig. 3(d)). The stoichiometry observed combined with the geometries observed in the AC-HRTEM images identified the material as $\left[\operatorname{Re}_{6} \mathrm{I}_{14}\right]^{2-}$ clusters which are structurally analogous to those previously discovered for Mo and $\mathrm{W}$ [31]. The structure of $\left[\operatorname{Re}_{6} \mathrm{I}_{14}\right]^{2-}$ can therefore be described as an octahedron of rhenium atoms, with capping iodide ligands on each face, and terminal iodides at each vertex (Figs. 3(e) and 3(f)). Structural models of the clusters were constructed and TEM simulations were conducted, the resultant images matched the observed projections (Figs. 3(b) and 3(c)) such as a cluster having a projected octahedral geometry. Upon prolonged imaging of the rhenium iodide clusters, the $60 \mathrm{kV}$ electron beam causes dimerisation and chain formation (Fig. 3(c)).

This is due to the electron beam rupturing a terminal Re-I bond, forming an unsaturated Re cation which must bond with a $\mu_{3}$-I from an adjacent cluster, resulting in the formation of polymeric iodide with a repeat unit of $\left[\operatorname{Re}_{6} \mathrm{I}_{12}\right]_{n}$. This is in contrast to $\left[\mathrm{W}_{6} \mathrm{I}_{14}\right]^{2-}$ that was reported to remain stable under the $80 \mathrm{kV}$ e-beam irradiation [31], which considering that constituent atoms in $\left[\operatorname{Re}_{6} \mathrm{I}_{14}\right]^{2-}$ receive less energy from the e-beam than in $\left[\mathrm{W}_{6} \mathrm{I}_{14}\right]^{2-}$ molecules, indicates the Re-I bond to be weaker than $\mathrm{W}-\mathrm{I}$.

\subsection{Reaction to form rhenium disulfide}

The reaction of the $\left[\operatorname{Re}_{6} I_{14}\right]^{2-}$ clusters with hydrogen sulfide gas produced nanoribbons of rhenium(IV) disulfide with lengths above $30 \mathrm{~nm}$ and width of approximately $1.3 \mathrm{~nm}$. The term nanoribbon is defined as a continuous quasi-1D material that has a width greater than its height resulting in a strip of material, which in this case is a single-layer of $\mathrm{ReS}_{2}$, with a width of four Re atoms. AC-HRTEM confirmed an ordered atomic structure and a twisted shape of the nanoribbon, whilst EDX analysis confirmed the presence of rhenium and sulfur in a $1: 3$ ratio (Figs. $4(\mathrm{a})$ and $4(\mathrm{e})$ ). The higher than expected amount of sulfur is most likely due to residual sulfur deposited in nanotubes during the reaction; also there was only a trace amount of iodine that remained in the system,
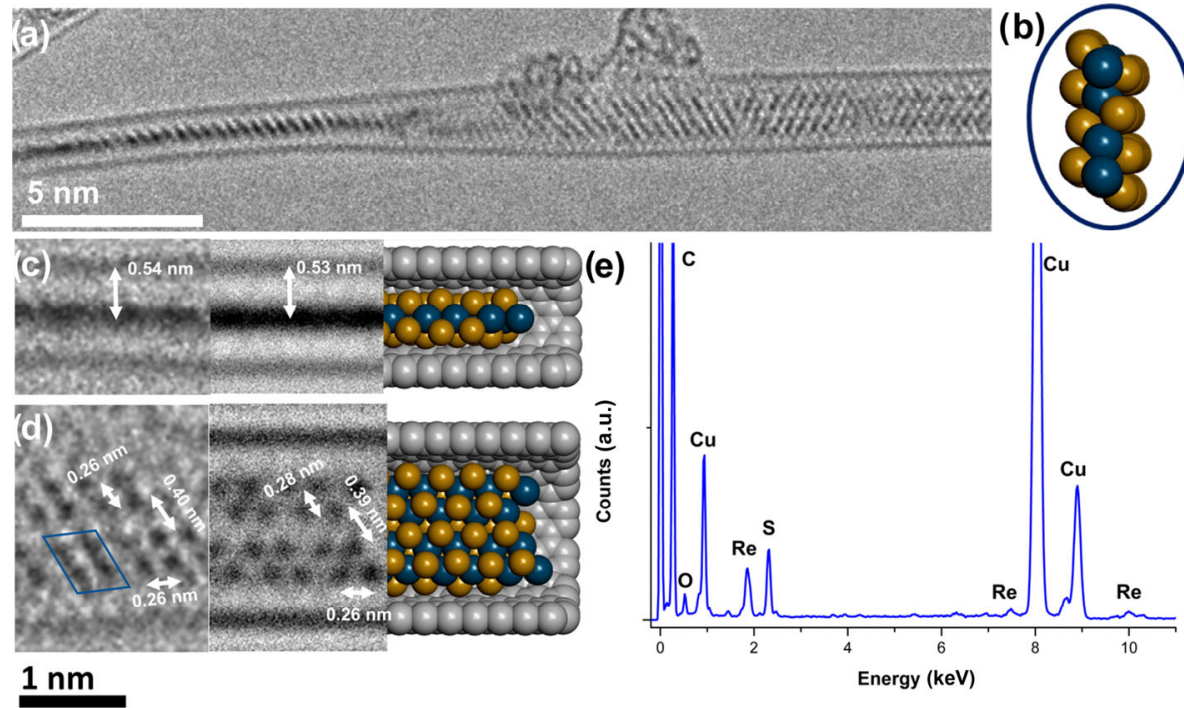

Figure 4 (a) $60 \mathrm{kV}$ AC-TEM image of a twisting rhenium disulfide nanoribbon inside a SWNT, ReS $@$ @WNT. (b) Structural model depicting the cross-section of the nanotube highlighting the radial deformation occurring in (a). (c) $60 \mathrm{kV}$ AC-HRTEM image annotated with the Re $\cdots \mathrm{C}$ interatomic distance; a HRTEM image simulation of an optimised structural model, showing the $\mathrm{ReS}_{2}$ in an edge-on orientation. (d) $60 \mathrm{kV}$ AC-HRTEM annotated with Re ${ }^{-*}$ Re interatomic distances and a parallelogram to illustrate the $\mathrm{Re}_{4}$ structural units; a TEM image simulation of an optimised structural model, showing the ReS $S_{2}$ nanoribbon in a face-on orientation. (e) EDX spectrum identifying Re and S present in the sample. The presence of copper is due to the TEM grid support. 
most likely in the form of polyiodides, which signified the near completion of the reaction. The Re:C ratio was found to be $1: 94$, very close to the content of rhenium in nanotubes in the first step of the synthesis (Section 3.2) which indicates no loss of encapsulated material in the subsequent reactions and a high yield of rhenium disulfide, correlating well with TEM images of $\mathrm{ReS}_{2} @ S W N T$. XPS analysis measured the binding energy of the $\operatorname{Re} 4 \mathrm{f}_{5 / 2}$ and $4 \mathrm{f}_{7 / 2}$ peaks to be at 44.3 and $41.9 \mathrm{eV}$ respectively (Fig. 2(c) and Fig. $S 4(a)$ in the ESM). The $4 \mathrm{f}_{7 / 2}$ value is slightly shifted compared to a previous study $(41.5 \mathrm{eV})$ which may be explained by a confinement effect [39]. The sulfur $2 \mathrm{p}_{3 / 2}$ and $2 \mathrm{p}_{1 / 2}$ peaks were located at 164.3 and $165.5 \mathrm{eV}$ respectively (Fig. S4(b) in the ESM). Raman spectroscopy analysis showed a small blue shift of the G-band compared to empty SWNTs, and a small blue shift compared to the encapsulated rhenium iodide species, suggesting the $\mathrm{ReS}_{2}$ nanoribbon is accepting electron density from the nanotubes (Fig. 2(d) and Table S1 in the ESM).

Due to the large difference between the atomic numbers of rhenium $(Z=75)$ and sulfur $(Z=16)$, only rhenium atoms were observed in the bright-field AC-HRTEM image as the singleatom contrast is proportional to the square root of the atomic number of an element. However, the nanotube sidewall can also be observed, despite the low atomic number of carbon $(Z=6)$, due to the overlap of approximately four atoms of carbon in projection at the SWNT sidewall (a thickness contrast). On the left-hand side of the AC-HRTEM image of ReS ${ }_{2} @ S W N T$ the nanoribbon is in an edge-on orientation in which the contrast is controlled by the thickness of the nanoribbon and the atoms overlapping in projection (Fig. 4(c)). The atoms in the edge-on projection are separated by a distance of $0.29 \mathrm{~nm}$. On the right-hand side the image displays clearly the face-on projection, which consists of each Re atom forming three types of distinct $\operatorname{Re} \cdots \operatorname{Re}$ distances resulting in the establishment of $\mathrm{Re}_{4}$ parallelograms, a distinct feature of the distorted $1 \mathrm{~T}$ structure (Fig. 4(d)). The nanoribbon is four rhenium atoms thick which equates to two rows of parallelograms. Each $\mathrm{Re}$ is bonded to six sulfur atoms in octahedral geometry and the sulfur edges are in contact with the nanotube sidewall. The $\mathrm{Re}_{4}$ parallelograms have a measured distance of $0.26 \mathrm{~nm}$ between adjacent rhenium atoms, whilst the two rows of parallelograms are separated by a distance of $0.40 \mathrm{~nm}$. These values are in good agreement with those from the structural model of the nanoribbon derived from the bulk $\mathrm{ReS}_{2}$ unit cell (Figs. 4(c) and $4(\mathrm{~d})$ ).

AC-HRTEM identified not only the structure of the nanoribbon but also the striking elliptical distortion of the SWNT host as a result of both shrinkage and expansion in response to orientation of guest-nanoribbon. The cross-section of the SWNT was measured to be a $1.1 \mathrm{~nm} \times 1.8 \mathrm{~nm}$ ellipse, and the change in projected nanotube cross-section is perfectly matched with the twisting of the rhenium sulfide nanoribbon (Fig. 4(b)). This demonstrates the strong synergy between the nanoribbon and the nanotube, similar to previously observed for $\mathrm{MoS}_{2} @ S W N T s$, as well as helical $\mathrm{CoI}_{2} @$ SWNT $[40,41]$.

\section{Conclusions}

Rhenium(IV) disulfide is an extraordinary transition metal dichalcogenide that retains its bulk electronic properties when exfoliated to a monolayer. Moreover, previous computational studies have shown that ultrathin nanoribbons also retain these properties and could therefore lead to being used in nanoscale electronic devices.

In this study, we synthesised ultrathin $\mathrm{ReS}_{2}$ nanoribbons using the extreme confinement within SWNT nano test tubes. The insertion of the initial source of rhenium, dirhenium decacarbonyl, was shown to be aided by van der Waals interactions indicated by the G-band not shifting position in the corresponding Raman spectra. The second step involved the transformation of dirhenium decacarbonyl into rhenium iodide clusters, $n\left[\operatorname{Re}_{6} \mathrm{I}_{14}{ }^{2-}\right] @ S W N T^{2 n+}$, which are described here for the first time. A combination of aberration-corrected TEM and EDX found these clusters were isostructural with W analogues reported inside SWNTs previously; however, the Re-I bonds appear to be more reactive than the W-I under the electron beam which resulted in partial oligomerisation of rhenium iodide clusters to $\left[\operatorname{Re}_{6} \mathrm{I}_{12}\right]_{n}$.

The reaction of the rhenium iodide clusters with hydrogen sulfide resulted in the formation of $\mathrm{ReS}_{2}$ nanoribbons of $1.3 \mathrm{~nm}$ in width. A radial deformation of the SWNTs occurred to accommodate the twisted $\mathrm{ReS}_{2}$ nanoribbons in order to maximise the interactions of the surface of the nanoribbons with the interior concave of the nanotubes. The nanoribbons were found to have the same distorted 1T structure as per the bulk material, shown by the presence of $\operatorname{Re}_{4}$ parallelograms. The synthesis within SWNTs was shown to be successful for the construction of ultrathin $\mathrm{ReS}_{2}$ nanoribbons owing to the precise control of reactions within SWNTs. As the resultant $\mathrm{ReS}_{2}$ nanoribbons are expected to retain their bulk electronic properties, including the direct bandgap, and being encapsulated within electrically conducting SWNT, ReS $2 @ S W N T$ hybrid materials may find future applications as nano-electrodes.

\section{Acknowledgements}

L. T. N. and A. N. K. are grateful to the Nanoscale and Microscale Research Centre (nmRC) for access to equipment, as well as to the Engineering and Physical Sciences Research Council (EPSRC) and the University of Nottingham for funding. J.B. and U.K. are grateful for the financial support of the German Research Foundation (DFG) (No. 424798828).

Electronic Supplementary Material: Supplementary material (including high resolution XPS scans, Raman shift positions and spectra) is available in the online version of this article at https://doi.org/10.1007/s12274-021-3650-2.

Open Access This article is licensed under a Creative Commons Attribution 4.0 International License, which permits use, sharing, adaptation, distribution and reproduction in any medium or format, as long as you give appropriate credit to the original author(s) and the source, provide a link to the Creative Commons licence, and indicate if changes were made.

The images or other third party material in this article are included in the article's Creative Commons licence, unless indicated otherwise in a credit line to the material. If material is not included in the article's Creative Commons licence and your intended use is not permitted by statutory regulation or exceeds the permitted use, you will need to obtain permission directly from the copyright holder.

To view a copy of this licence, visit http://creativecommons.org/licenses/by/4.0/.

\section{References}

[1] Rahman, M.; Davey, K.; Qiao, S. Z. Advent of 2D rhenium disulfide $\left(\mathrm{ReS}_{2}\right)$ : Fundamentals to applications. Adv. Funct. Mater. 2017, 27, 1606129.

[2] Liu, E. F.; Fu, Y. J.; Wang, Y. J.; Feng, Y. Q.; Liu, H. M.; Wan, X. G.; Zhou, W.; Wang, B. G.; Shao, L. B.; Ho, C. H. et al. Integrated digital inverters based on two-dimensional anisotropic $\mathrm{ReS}_{2}$ field-effect 
transistors. Nat. Commun. 2015, 6, 6991.

[3] Feng, Y. Q.; Zhou, W.; Wang, Y. J.; Zhou, J.; Liu, E. F.; Fu, Y. J.; Ni, Z. H.; Wu, X. L.; Yuan, H. T.; Miao, F. et al. Raman vibrational spectra of bulk to monolayer $\mathrm{ReS}_{2}$ with lower symmetry. Phys. Rev. $B$ 2015, 92, 054110.

[4] Tongay, S.; Sahin, H.; Ko, C.; Luce, A.; Fan, W.; Liu, K.; Zhou, J.; Huang, Y. S.; Ho, C. H.; Yan, J. Y. et al. Monolayer behaviour in bulk $\mathrm{ReS}_{2}$ due to electronic and vibrational decoupling. Nat. Commun. 2014, 5, 3252.

[5] Splendiani, A.; Sun, L.; Zhang, Y. B.; Li, T. S.; Kim, J.; Chim, C. Y.; Galli, G; Wang, F. Emerging photoluminescence in monolayer $\mathrm{MoS}_{2}$. Nano Lett. 2010, 10, 1271-1275.

[6] Zhao, W. J.; Ghorannevis, Z.; Chu, L. Q.; Toh, M.; Kloc, C.; Tan, P. H.; Eda, G. Evolution of electronic structure in atomically thin sheets of $\mathrm{WS}_{2}$ and $\mathrm{WSe}_{2}$. ACS Nano 2013, 7, 791-797.

[7] Murray, H. H.; Kelty, S. P.; Chianelli, R. R.; Day, C. S. Structure of rhenium disulfide. Inorg. Chem. 1994, 33, 4418-4420.

[8] Chenet, D. A.; Aslan, O. B.; Huang, P. Y.; Fan, C.; van der Zande, A. M.; Heinz, T. F.; Hone, J. C. In-plane anisotropy in mono- and few- layer $\mathrm{ReS}_{2}$ probed by Raman spectroscopy and scanning transmission electron microscopy. Nano Lett. 2015, 15, 5667-5672.

[9] Brorson, M.; Hansen, T. W.; Jacobsen, C. J. H. Rhenium(IV) sulfide nanotubes. J. Am. Chem. Soc. 2002, 124, 11582-11583.

[10] Tu, W. X.; Denizot, B. Synthesis of small-sized rhenium sulfide colloidal nanoparticles. J. Colloid Interface Sci. 2007, 310, 167170.

[11] An, Q. W.; Liu, Y.; Jiang, R. J.; Meng, X. Q. Chemical vapor deposition growth of $\mathrm{ReS}_{2}$ nanowires for high-performance nanostructured photodetector. Nanoscale 2018, 10, 14976-14983.

[12] He, X. X.; Liu, F. C.; Hu, P.; Fu, W.; Wang, X. L.; Zeng, Q. S.; Zhao, W.; Liu, Z. Chemical vapor deposition of high-quality and atomically layered $\mathrm{ReS}_{2}$. Small 2015, 11, 5423-5429.

[13] Yu, Z. G.; Cai, Y. Q.; Zhang, Y. W. Robust direct bandgap characteristics of one- and two-dimensional $\mathrm{ReS}_{2}$. Sci. Rep. 2015, 5, 13783.

[14] Wilder, J. W. G.; Venema, L. C.; Rinzler, A. G.; Smalley, R. E.; Dekker, C. Electronic structure of atomically resolved carbon nanotubes. Nature 1998, 391, 59-62.

[15] Iijima, S.; Ichihashi, T. Single-shell carbon nanotubes of 1-nm diameter. Nature 1993, 363, 603-605.

[16] Bethune, D. S.; Kiang, C. H.; de Vries, M. S.; Gorman, G.; Savoy, R.; Vazquez, J.; Beyers, R. Cobalt-catalysed growth of carbon nanotubes with single-atomic-layer walls. Nature 1993, 363, 605-607.

[17] Sloan, J.; Grosvenor, S. J.; Friedrichs, S.; Kirkland, A. I.; Hutchison, J. L.; Green, M. L. H. A one-dimensional $\mathrm{BaI}_{2}$ chain with five- and sixcoordination, formed within a single-walled carbon nanotube. Angew. Chem., Int. Ed. 2002, 41, 1156-1159.

[18] Slade, C. A.; Sanchez, A. M.; Sloan, J. Unprecedented new crystalline forms of $\mathrm{SnSe}$ in narrow to medium diameter carbon nanotubes. Nano Lett. 2019, 19, 2979-2984.

[19] Smith, B. W.; Monthioux, M.; Luzzi, D. E. Encapsulated $C_{60}$ in carbon nanotubes. Nature 1998, 396, 323-324.

[20] Allen, C. S.; Ito, Y.; Robertson, A. W.; Shinohara, H.; Warner, J. H. Two-dimensional coalescence dynamics of encapsulated metallofullerenes in carbon nanotubes. ACS Nano 2011, 5, 10084-10089.

[21] Sloan, J.; Novotny, M. C.; Bailey, S. R.; Brown, G.; Xu, C.; Williams, V. C.; Friedrichs, S.; Flahaut, E.; Callender, R. L.; York, A. P. E. et al. Two layer 4:4 co-ordinated KI crystals grown within single walled carbon nanotubes. Chem. Phys. Lett. 2000, 329, 61-65.

[22] Bendall, J. S.; Ilie, A.; Welland, M. E.; Sloan, J.; Green, M. L. H. Thermal stability and reactivity of metal halide filled single-walled carbon nanotubes. J. Phys. Chem. B 2006, 110, 6569-6573.

[23] Eliseev, A. A.; Yashina, L. V.; Brzhezinskaya, M. M.; Chernysheva, M. V.; Kharlamova, M. V.; Verbitsky, N. I.; Lukashin, A. V.; Kiselev, N. A.; Kumskov, A. S.; Zakalyuhin, R. M. et al. Structure and electronic properties of $\mathrm{AgX}(x=\mathrm{Cl}, \mathrm{Br}, \mathrm{I})$-intercalated single-walled carbon nanotubes. Carbon 2010, 48, 2708-2721.

[24] Costa, P. M. F. J.; Sloan, J.; Rutherford, T.; Green, M. L. H. Encapsulation of $\operatorname{Re}_{x} \mathrm{O}_{y}$ clusters within single-walled carbon nanotubes and their in tubulo reduction and sintering to Re metal. Chem. Mater. 2005, 17, 6579-6582.
[25] Friedrichs, S.; Sloan, J.; Green, M. L. H.; Hutchison, J. L.; Meyer, R. R.; Kirkland, A. I. Simultaneous determination of inclusion crystallography and nanotube conformation for a $\mathrm{Sb}_{2} \mathrm{O}_{3} /$ single-walled nanotube composite. Phys. Rev. B 2001, 64, 045406.

[26] Wang, J. W.; Kuimova, M. K.; Poliakoff, M.; Briggs, G. A. D.; Khlobystov, A. N. Encapsulation and IR probing of cube-shaped octasilasesquioxane $\mathrm{H}_{8} \mathrm{Si}_{8} \mathrm{O}_{12}$ in carbon nanotubes. Angew. Chem., Int. Ed. 2006, 45, 5188-5191.

[27] Stoppiello, C. T.; Biskupek, J.; Li, Z. Y.; Rance, G. A.; Botos, A.; Fogarty, R. M.; Bourne, R. A.; Yuan, J.; Lovelock, K. R. J.; Thompson, P. et al. A one-pot-one-reactant synthesis of platinum compounds at the nanoscale. Nanoscale 2017, 9, 14385-14394.

[28] Chuvilin, A.; Bichoutskaia, E.; Gimenez-Lopez, M. C.; Chamberlain, T. W.; Rance, G. A.; Kuganathan, N.; Biskupek, J.; Kaiser, U.; Khlobystov, A. N. Self-assembly of a sulphur-terminated graphene nanoribbon within a single-walled carbon nanotube. Nat. Mater. 2011, 10, 687692.

[29] Eliseev, A. A.; Chernysheva, M. V.; Verbitskii, N. I.; Kiseleva, E. A.; Lukashin, A. V.; Tretyakov, Y. D.; Kiselev, N. A.; Zhigalina, O. M.; Zakalyukin, R. M.; Vasiliev, A. L. et al. Chemical reactions within single-walled carbon nanotube channels. Chem. Mater. 2009, 21, 5001-5003.

[30] Wang, Z. Y.; Zhao, K. K.; Li, H.; Liu, Z.; Shi, Z. J.; Lu, J.; Suenaga, K.; Joung, S. K.; Okazaki, T.; Jin, Z. X. et al. Ultra-narrow $\mathrm{WS}_{2}$ nanoribbons encapsulated in carbon nanotubes. J. Mater. Chem. 2011, $21,171-180$.

[31] Botos, A.; Biskupek, J.; Chamberlain, T. W.; Rance, G. A.; Stoppiello, C. T.; Sloan, J.; Liu, Z.; Suenaga, K.; Kaiser, U.; Khlobystov, A. N. Carbon nanotubes as electrically active nanoreactors for multi-step inorganic synthesis: Sequential transformations of molecules to nanoclusters and nanoclusters to nanoribbons. J. Am. Chem. Soc. 2016, 138, 8175-8183.

[32] Qi, F.; He, J. R.; Chen, Y. F.; Zheng, B. J.; Li, Q.; Wang, X. Y.; Yu, B.; Lin, J.; Zhou, J. H.; Li, P. J. et al. Few-layered $\operatorname{ReS}_{2}$ nanosheets grown on carbon nanotubes: A highly efficient anode for highperformance lithium-ion batteries. Chem. Eng. J. 2017, 315, 10-17.

[33] Linck, M.; Hartel, P.; Uhlemann, S.; Kahl, F.; Müller, H.; Zach, J.; Haider, M.; Niestadt, M.; Bischoff, M.; Biskupek, J. et al. Chromatic aberration correction for atomic resolution TEM imaging from 20 to 80 kV. Phys. Rev. Lett. 2016, 117, 076101.

[34] Rez, D.; Rez, P.; Grant, I. Dirac-fock calculations of X-ray scattering factors and contributions to the mean inner potential for electron scattering. Acta Cryst. A 1994, 50, 481-497.

[35] Cao, K. C.; Chamberlain, T. W.; Biskupek, J.; Zoberbier, T.; Kaiser, U.; Khlobystov, A. N. Direct correlation of carbon nanotube nucleation and growth with the atomic structure of rhenium nanocatalysts stimulated and imaged by the electron beam. Nano Lett. 2018, 18, 6334-6339.

[36] Feltham, R. D.; Brant, P. XPS studies of core binding energies in transition metal complexes. 2. Ligand group shifts. J. Am. Chem. Soc. 1982, 104, 641-645.

[37] Grigorian, L.; Williams, K. A.; Fang, S.; Sumanasekera, G. U.; Loper, A. L.; Dickey, E. C.; Pennycook, S. J.; Eklund, P. C. Reversible intercalation of charged iodine chains into carbon nanotube ropes. Phys. Rev. Lett. 1998, 80, 5560-5563.

[38] Kharlamova, M. V. Electronic properties of single-walled carbon nanotubes filled with manganese halogenides. Appl. Phys. A 2016, $122,791$.

[39] Davis, S. M. Photoemission studies of rhenium disulfide oxidation: Altered core-level structure and reactivity of defect sites. Catal. Lett. 1989, 2, 1-7.

[40] Wang, Z. Y.; Li, H.; Liu, Z.; Shi, Z. J.; Lu, J.; Suenaga, K.; Joung, S. K.; Okazaki, T.; Gu, Z. N.; Zhou, J. et al. Mixed low-dimensional nanomaterial: 2D ultranarrow $\mathrm{MoS}_{2}$ inorganic nanoribbons encapsulated in quasi-1D carbon nanotubes. J. Am. Chem. Soc. 2010, 132, $13840-13847$.

[41] Philp, E.; Sloan, J.; Kirkland, A. I.; Meyer, R. R.; Friedrichs, S.; Hutchison, J. L.; Green, M. L. H. An encapsulated helical onedimensional cobalt iodide nanostructure. Nat. Mater. 2003, 2 , 788-791. 